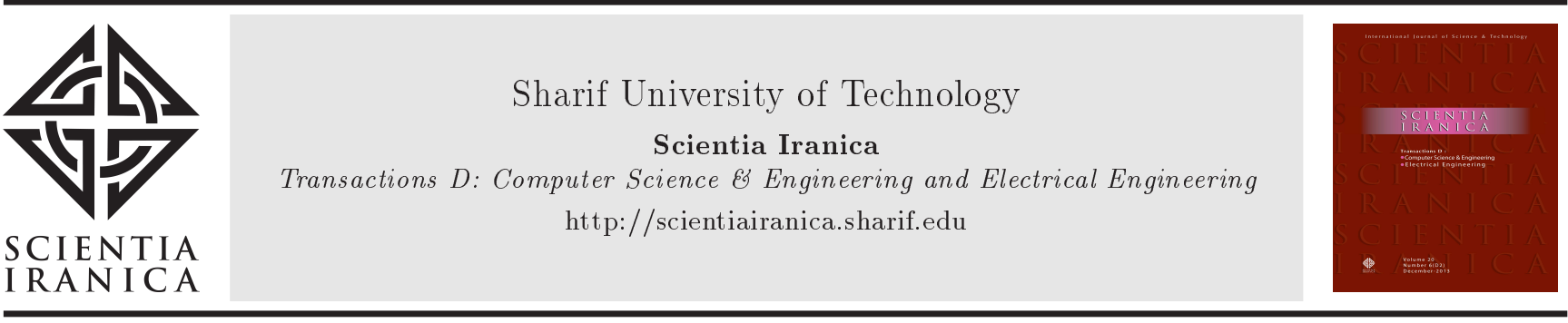

\title{
A new aspect of transition between line and continuous spectrum and its relation to seismic influence on structures
}

\author{
R. Babića ${ }^{a}$ L. Babić ${ }^{b}$, and B. Jakšićc ${ }^{a, *}$ \\ a. Department of Electrical and Computing Engineering, Faculty of Technical Sciences, University of Pristina, Knjaza Milosa \\ Street, No. 7, Kosovska Mitrovica, P.O. Box 38220, Serbia. \\ b. Department of Civil Engineering, Faculty of Technical Sciences, University of Pristina, Knjaza Milosa Street, No. 7 , Kosovska \\ Mitrovica, P.O. Box 38220, Serbia.
}

Received 4 August 2017; received in revised form 8 November 2017; accepted 23 April 2018

\section{KEYWORDS}

Line spectrum;

Fourier analysis;

Spectrum invariance;

Seismic signal;

Structure dynamics.

\begin{abstract}
This study considers a new view of the transition from periodic to aperiodic signals in time and spectral domains, thus pointing out the idea of how the concept of infinity is involved. The results of this paper contribute to a better understanding of the nature of both spectral descriptions and conditions of their practical use, particularly in unusual cases. Therefore, this study highlights invariance of convergence of spectrum by introducing some numerical parameters, which exactly describe such a process. Their behavior is numerically examined in detail. In addition, the opposite transition from aperiodic to periodic is considered to clarify the meaning of the spectral line. To suggest the applicability of our analysis, an actual seismic signal is used. By extracting the most prominent waveform part, regarding its influence on structures, a periodic signal is formed whose line spectrum can clearly show possible resonance with the vibrating tones of structures.
\end{abstract}

(C) 2020 Sharif University of Technology. All rights reserved.

\section{Introduction}

It is well known that the Fourier Series (FS) calculates the line spectrum of a periodic function instead of a periodic signal, since the latter cannot simply exist in the strict sense of the word, i.e., to be periodic over the whole $t$ domain, according to the FS definition. The periodic function is a mathematical representation of a periodic signal, which is, by itself, a version of the "periodic", or real, signal that is periodized to infinity.

\footnotetext{
*. Corresponding author. Tel.: +38 128425320

Fax: + 38 128425322

E-mail addresses: ranko.babic@pr.ac.rs (R. Babić);

lidija.babic@pr.ac.rs (L.Babić); branimir.jaksic@pr.ac.rs (B. Jakšić)
}

doi: $10.24200 /$ sci. 2018.20326
Although an entity is placed between periodic signal and its function, there are indeed different entities. The first entity is composed of pure numerical values, while the other one expresses the values of some physical quantities. As for the FS, it acts only in accordance with the given shape ([1], pp. 34-40). There are those who assign physical meaning to mathematical shapes and interpret the nature of the entity before and after it is subjected to the FS.

The term "line" stands for its discrete nature, originating from a suitable graphical representation. This term is of importance to our theoretical considerations.

Nevertheless, in scientific research and engineering practice, one often use line spectral representation of "periodic" signals because of its convenience in describing the actual issue. However, always, it 
should be noted how much a "periodic" signal differs from its aperiodic (theoretical) counterpart in order to appropriately explain the actual results.

Generally, signals that are not strictly periodic should be regarded as aperiodic ones, which, depending on their duration, are further divided into impulses and finite realizations of some processes (random, periodic, etc.). Whatever the actual duration of signal/function may be, it is always formally extended by zero to infinity to cover the whole $t$-axis/domain. For theoretical purposes, finite random realisations are often generalized to cover $t \in(-\infty,+\infty)$ or $t \in(-\infty, 0)$ [2]. Moreover, even a strict periodic signal can be formally treated as an aperiodic one if considered as a whole, not as a sequence of repeating waveforms ([1], pp. 119$133)$.

Herein, a practical rule of thumb is proposed on how to distinguish impulses from long-lasting aperiodic signals. It is based on the number of changes, i.e., fluctuations, in the signal duration. That is, an aperiodic signal may last long, yet can be considered as an impulse since it slowly fluctuates and comprises several changes.

Refining this rule, i.e., how much changes can differentiate impulses from the lasting aperiodic signals, calls for a deeper investigation; however, herein, this study considers acoustic, or much better seismic, waveforms to find a clue for such a recommendation.

Aperiodic signals are represented in the frequency domain by a continuous spectrum. The nature of the spectral density is important to better interpret research results.

The very transition between those two spectral entities, line and continuous, if properly considered, may give a deeper insight and improve one's understanding and even broaden the scope of waveform analysis. Seismogram analysts and station operators should possess the capability to "understand each wiggle" in a seismic record [3], especially in unusual cases. The same holds for some researchers and engineers that encounter seismic factors in their work. It is implied here that they should have clarified the notions of all aspects of the seismogram, including its treatment by Fourier tools. The FS calculates the spectrum of a periodic function (not of a periodic signal) since such a signal cannot simply exist in the strict sense of the word, i.e., to be periodic over the whole $t$ domain.

For a periodic time function $s(t)$, its spectral (Fourier) coefficients are easily calculated as follows:

$$
\begin{gathered}
S_{n}=S(n)=S\left(n \omega_{0}\right)=\frac{1}{T} \int_{-T / 2}^{T / 2} s(t) e^{-i\left(n \omega_{0}\right) t} d t, \\
n=0, \pm 1, \pm 2, \cdots,
\end{gathered}
$$

which covers the infinite $\omega$ domain and is readily seen if Eq. (1) is rewritten in the following:

$$
\begin{gathered}
S_{n}=S(n)=S\left(n \omega_{0}\right)=\frac{1}{T} \int_{k T}^{(k+1) T} s(t) e^{-i\left(n \omega_{0}\right) t} d t \\
n=0, \pm 1, \pm 2, \cdots, \\
k=0, \pm 1, \pm 2, \cdots,
\end{gathered}
$$

where it is observed how the FS maps the whole $t$ domain to the whole $\omega$ domain.

Expression (2) gives an implication of $t$ domain infinity such that the same operation is carried out in each period throughout $t$ domain, i.e., one actually deals with a single period, yet treats it like dealing with all others in the same manner. Therefore, possible mistakes and errors are predicted while calculating signal's spectrum. If not, the computer program does not know how many periods there are.

For a time process (time variations of some physical quantity), described by signal $\sigma(t)$, which is of finite duration:

$$
\exists \sigma(t) \quad \forall t \in\left(t_{1}, t_{2}\right),
$$

its continuous spectrum is determined by the Fourier Transform (FT), encompassing the whole $t$ domain:

$$
S(\omega)=\int_{-\infty}^{\infty} s(t) e^{-i \omega t} d t \quad \text { i.e. } \int_{-\infty}^{\infty}[] d t
$$

As mentioned before, prior to applying the FT to $\sigma(t)$, it must be expressed by function $s(t)$, extending its definition domain to the whole $t$-axis as in the following:

$$
\begin{aligned}
& \exists s(t) \quad \forall t \in(-\infty,+\infty), \\
& \sigma(t) \equiv s(t) \quad \forall t \in\left(t_{1}, t_{2}\right),
\end{aligned}
$$

ensuring that there is no other signal on the rest of $t$ axis. This is not hair-splitting process as it may seem, yet the need to follow fundamental issues to prevent principal mistakes is implied.

Another infinite domain, frequency, is hidden in the FT expression:

$$
\begin{aligned}
& S(\omega)=\int_{-\infty}^{\infty} s(t) e^{-i \omega t} d t \\
&-\infty<t<\infty-\infty<\omega<\infty .
\end{aligned}
$$

The result of the performance of the FT on $s(t)$ is a continuous spectrum $S(\omega)$.

In the following, the relationship between these two spectral entities is examined. 


\section{The essence of the relationship between FS and FT}

Making an aperiodic signal (function) $s_{A}(t)$ from its periodic counterpart $s_{P}(t)$ is seemingly simple (Figure 1). Let us assume:

$$
s_{A}(t)=s_{P}(t) \quad 0 \leq t<T .
$$

In other words:

$$
\begin{aligned}
s_{P}(t) & =\sum_{n=-\infty}^{+\infty} S_{n}^{P} e^{i n \omega_{0} t} \\
& =S_{0}+\sum_{n=1}^{+\infty} 2\left|S_{n}^{P}\right| \cos \left[n \omega_{0} t+\theta(\omega)\right] \\
& =s_{A}(t) \quad 0<t<T,
\end{aligned}
$$

where all harmonics outside the interval $[0, T]$ are cut off. This operation in $t$ domain causes drastic mathematical disruption since it disintegrates the nature of trigonometric function (Figure 2) by contradicting the very definition of FS based directly on the space of trigonometric functions, which are inherently infinite, covering the whole $t$ domain ([1], pp. 87-94).

To find a mathematically proper way to relate periodic and aperiodic functions/signals, the limit process is addressed:

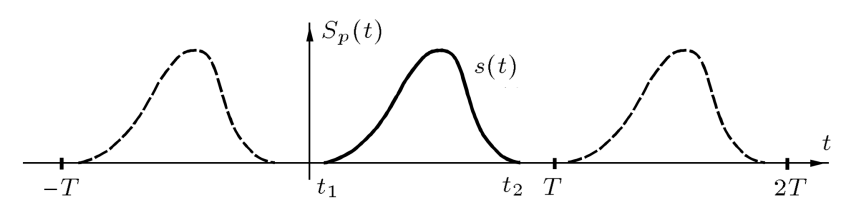

Figure 1. Aperiodic function derived from the corresponding periodic signal by simply considering one period content.
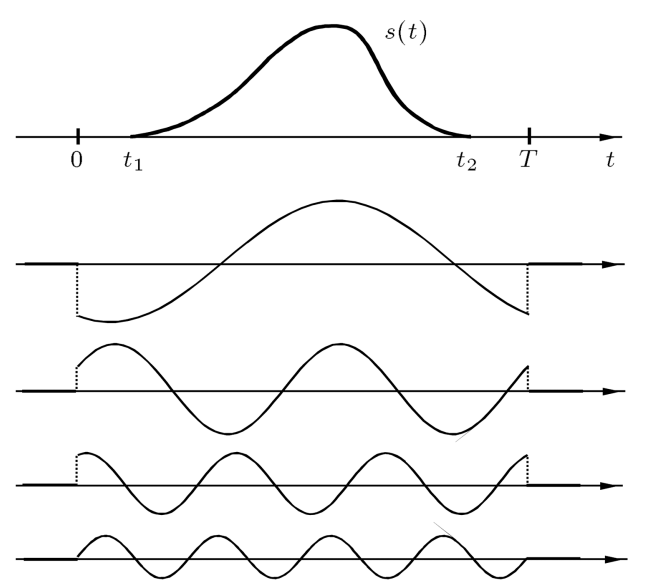

Figure 2. Creating an aperiodic signal by cutting off one period from its periodic counterpart so that all trigonometric functions involved in the periodic signal description are cut off.

$$
s_{P}(t) \underset{T \rightarrow \infty}{\longrightarrow} s_{A}(t) .
$$

Considering that spectral analysis, e.g., Yarlagadda [4], is well and widely known throughout classical literature, let us follow this limit process by formulae.

Considering Eqs. (1) and (8), we write:

$$
\begin{aligned}
& s_{P}(t)=\sum_{n=-\infty}^{\infty}\left[\frac{1}{T} \int_{0}^{T} s_{P}(x) e^{-i n \omega_{0} x} d x\right] e^{-i n \omega_{0} t} \\
& =\frac{1}{2 \pi} \sum_{n=-\infty}^{\infty}\left[\int_{0}^{T} s_{P}(x) e^{-i n \omega_{0} x} d x\right] e^{-i n \omega_{0} t} \omega_{0} \\
& \underset{\substack{T \rightarrow \infty \\
n \omega_{0} \rightarrow \omega \\
\omega_{0} \rightarrow d \omega}}{\longrightarrow} \frac{1}{2 \pi} \int_{-\infty}^{\infty} e^{i \omega t}\left(\int_{t_{1}}^{t_{2}} s(x) e^{-i \omega t} d x\right) d \omega \\
& =\frac{1}{2 \pi} \int_{-\infty}^{\infty} S(\omega) e^{i \omega t} d \omega=s_{A}(t) .
\end{aligned}
$$

Herein, pulse definition interval $\left(t_{1}, t_{2}\right)$ to $(-\infty,+\infty)$ is simply extended. Now, the formal analogy in these two expressions is highlighted as follows:

$$
s_{P}(t)=\sum_{n=-\infty}^{\infty} S_{n}^{P} e^{i n \omega_{0} t}=\sum_{n=-\infty}^{\infty}\left|S_{n}^{P}\right| e^{i\left[n \omega_{0} t+\theta_{n}\right]},
$$

and:

$$
\begin{aligned}
s_{A}(t) & =\frac{1}{2 \pi} \int_{-\infty}^{\infty} S_{A}(\omega) e^{i \omega t} d \omega \\
& =\int_{-\infty}^{\infty}\left[\frac{1}{2 \pi}\left|S_{A}(\omega)\right| d \omega\right] e^{i[\omega t+\theta(\omega)]},
\end{aligned}
$$

which define conventional and infinitesimal series, respectively.

Each spectral component has an infinitesimal amplitude:

$$
d A_{(\omega)}=\frac{1}{2 \pi}\left|S_{A}(\omega)\right| \cdot d \omega
$$

where the frequency differs infinitesimally from frequencies of neighboring ones. As a measure of difference, $d \omega$ can be used.

If the progress of the limit process in both $t$ and $\omega$ domains is considered, we notice that as pulses of $s_{P}(t)$ spread out, amplitudes of spectral components become denser and smaller (Figure 3 ) through several steps and in the case of periodic rectangular pulses for the sake of clarification. When the neighboring pulses become 


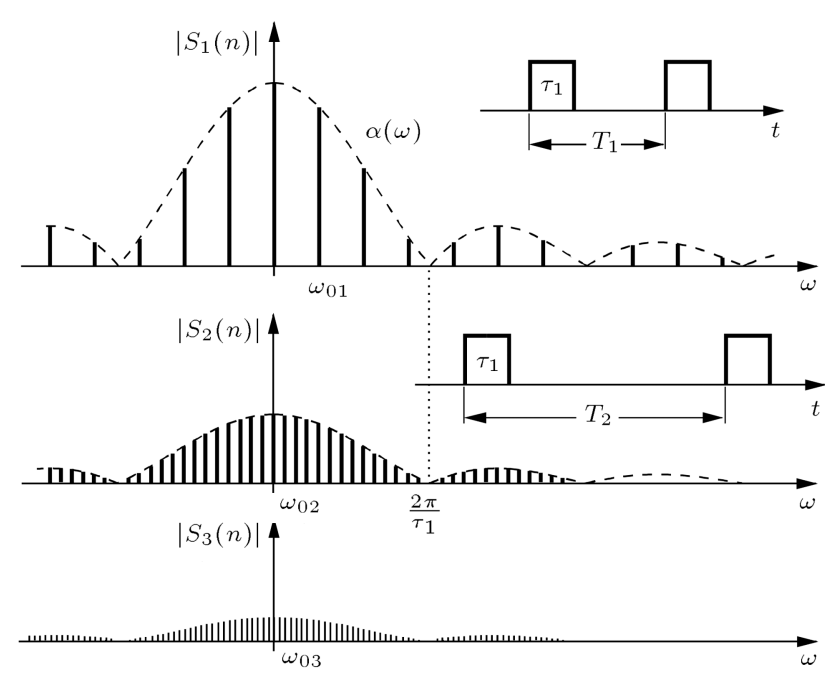

Figure 3. The spectrum becoming denser and component amplitudes smaller as pulses in periodic function spread out.

infinitely far away and no one can determine how infinitely far they are, components become infinitely small and dense. Apparently, the (line) spectrum disappears, and Eq. (10) does not fit the situation on the terrain. These interrelated aspects of infinity are very interesting: when neighboring pulses stand infinitely far away, it can be concluded that the whole $t$ domain is at disposal to a single pulse ([1] pp. 9394 ), making it a truly aperiodic one and preparing it for correct action of the FT in accordance with its definition.

Therefore, if we seek to capture a useful picture of the spectrum function, which seemingly disappears, we must get a number of appropriate valuable characteristics to override infinitesimal ambiguity. It is a wellknown method for comparing one infinitesimal with another, or so-called normalization:

$$
\frac{|S(\omega)| d \omega}{|S(0)| d \omega}=K(\omega) .
$$

Therefore, the continuous spectrum shows relations between amplitudes of components instead of their actual values which one gets by multiplying spectrum (function) values by $d \omega$ (Eq. (13)). Hence, values of $|S(\omega)|$ show the participation of components in creating the signal waveform (its $t$ function).

\section{Numerical results and discussion}

Another interpretation involves the density of spectral components, i.e., harmonics, so-called microharmonics, expressed by the rectangular area (Figure 4) ([1], pp. 91-92):

$$
P_{\Delta}=\left|S_{\Delta}(\omega)\right| \cdot \Delta \omega=\sum_{n \in \Delta \omega} A_{n} .
$$

Let us define a quantity:

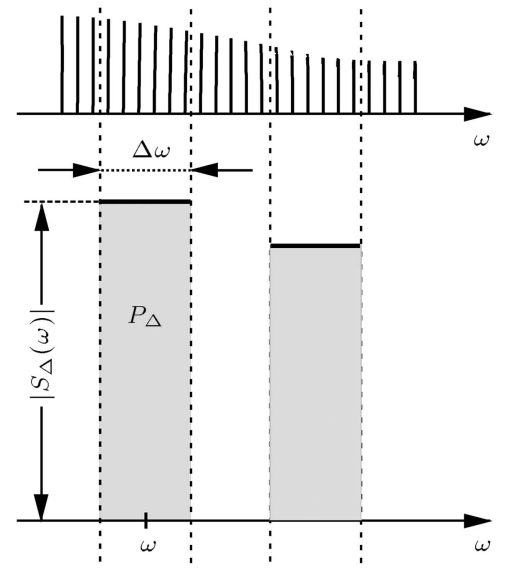

Figure 4. Sum of amplitudes of harmonics, which belong to the frequency interval, represented by the area of the corresponding rectangle.

$$
\sum_{n \omega_{0} \in \delta \omega}\left|S\left(n \omega_{0}\right)\right|
$$

which is the sum of the amplitudes of all components within a frequency interval $\delta \omega$, and the change of its value is considered during the limit process. Hence, for $T=T_{1}$, we obtain:

$$
\Sigma_{1}=\sum_{n \omega_{0} \in \delta \omega}\left|S^{(1)}\left(n \omega_{0}\right)\right|,
$$

for $T=T_{2}$, we have:

$$
\Sigma_{2}=\sum_{n \omega_{0} \in \delta \omega}\left|S^{(2)}\left(n \omega_{0}\right)\right|,
$$

etc. Sums $\Sigma_{1}, \Sigma_{2}, \Sigma_{3}, \ldots$ are usually different because of the discrete nature of spectrum (see Figures 3 or 4) and form a sequence which is not monotonic necessarily; however, it converges to an asymptotic value for $T \rightarrow \infty$.

In this case, we see how "mathematical liquid" of components is not compressible and does not disappear at $T \rightarrow \infty$.

A calculator (Windows XP) and a simple $(\mathrm{C}++)$ program are used to carry out calculations to examine the behavior of the quoted parameters during the limit process. Any programming language can be used for this purpose. A periodic train of rectangular pulses, as shown in Figure 3, with parameters $E=1 \mathrm{~V}, \tau=$ $1 \mathrm{~ms}$, is considered. First, zero of amplitude spectrum envelope is measured in $(\mathrm{krad} / \mathrm{s})$ as in the following:

$$
\omega_{Z}=\frac{2 \pi}{\tau}=6.28318530718 .
$$

The line spectrum changes in the frequency interval are examined as follows:

$$
\Delta \omega=\left[3 \cdot \frac{\omega_{Z}}{6} ; 4 \cdot \frac{\omega_{Z}}{6}\right] .
$$

With respect to the effect of the coincidence caused by 
harmonic relations between component frequencies, we made corrections and, finally, defined the interval as follows:

$$
\Delta \omega=\left[2.9 \cdot \frac{\omega_{Z}}{6} ; \quad 4.1 \cdot \frac{\omega_{Z}}{6}\right] .
$$

Accordingly, uncertainty in calculating the sum (16) is avoided when the frequency of a certain harmonic coincides with an interval border. For example, in the case of $T_{11}=60 \tau, 29 \mathrm{th}$ and 41st harmonics coincide with interval borders (Eq. (21)). Therefore, the values of 2.91 and 4.09 may be highly appropriate for interval borders since the coincidence appears to be far at 291st and 409th harmonics. However, the error in amplitude summation (16) is not so critical. Amplitude is calculated as follows:

$$
A_{n}=\frac{1}{T}\left|\frac{\sin (n \pi / T)}{n \pi / T}\right|,
$$

and is measured in [V].

Calculated parameters are given in Table 1 (rounded off to 10 decimals).

Therefore, the calculation interval, at which we will trace the spectrum change, is:

$$
\begin{aligned}
& \Delta \omega\left[\omega_{\min } ; \omega_{\max }\right] \\
& =[3.03687289847033 ; 4.29350995990633] \\
& \quad=D w=\left[D w_{\min } ; D w_{\max }\right] .
\end{aligned}
$$

In the following, this study presents several calculation steps (and use, for convenience, normal variables instead of italic style for variables).

Table 1. Calculation parameters.

\begin{tabular}{cll}
\hline Envelope zero & $w_{Z}=2 \cdot \pi / \tau$ & 6.2831853072 \\
& $w_{Z} / 6$ & 1.0471975512 \\
Low border & $2.9 \cdot w_{Z} / 6$ & 3.0368728985 \\
Up border & $4.1 \cdot w_{Z} / 6$ & 4.29350995991 \\
\hline
\end{tabular}

For $T_{1}=4 \cdot \tau$ (simply meaning that the period is 4 times the pulse duration, which is a good relative measure for $T$ ), the fundamental frequency (first harmonic) is $w_{01}=2 \pi / T_{1}=1.5707963268$, and only the second harmonic belongs to the interval $\Delta \omega=\left[\omega_{\min } ; \omega_{\max }\right]$, since $w_{21}=2 \cdot w_{01}=3.1415926536 \in \mathrm{Dw}$. Its amplitude is $A_{21}=1 / T_{1} \cdot\left|\sin \left(2 \cdot \pi / T_{1}\right) /\left(2 \cdot \pi / T_{1}\right)\right|=$ 0.1591549431 , and the $\operatorname{sum}(16)$ is sum $1=A_{21}=$ 0.1591549431 .

For $T_{4}=16 \cdot \tau$, results are given in Table 2 .

The rest of the manual calculations is given in Table 3 up to $T_{13}=80 \cdot \tau$ (rounded off to 4 decimals).

The label $N_{\text {har }}$ stands for the number of encompassed harmonics within interval $D w$.

To illustrate the problem of coincidence, detailed calculations (by Windows XP calculator, at full precision) are presented for the critical value of $T_{11}=60 \tau$.

The fundamental frequency is:

$$
\begin{aligned}
W_{011} & =2 \cdot \pi / T_{11} \\
& =0.10471975511965977461542144610
\end{aligned}
$$

\begin{tabular}{|c|c|c|c|c|c|}
\hline Harmonic & Frequency & $\begin{array}{c}\text { Frequency } \\
\text { value }\end{array}$ & $\begin{array}{c}\text { Bel. } \\
\text { to } D w\end{array}$ & $\begin{array}{l}\text { Harmonic } \\
\text { amplitude }\end{array}$ & $\begin{array}{c}\text { Amplitude } \\
\text { value }\end{array}$ \\
\hline 1 st & $w_{04}=2 \cdot \pi / T \tau$ & 0.3926990817 & & // & \\
\hline 7 th & $7 \cdot w_{04}$ & 2.7488935718 & $\notin D w$ & $/ /$ & \\
\hline 8 th & $8 \cdot w_{04}$ & 3.1415926535 & $\in D w$ & $A_{84}=1 / T_{4} \cdot\left|\sin \left(8 \cdot \pi / T_{4}\right) /\left(8 \cdot \pi / T_{4}\right)\right|$ & 0.0397887358 \\
\hline 9 th & $9 \cdot w_{04}$ & 3.5342917352 & $\in D w$ & $A_{94}=1 / T_{4} \cdot\left|\sin \left(9 \cdot \pi / T_{4}\right) /\left(9 \cdot \pi / T_{4}\right)\right|$ & 0.0346881834 \\
\hline 10 th & $10 \cdot w_{04}$ & 3.9269908169 & $\in D w$ & $A_{104}=1 / T_{4} \cdot\left|\sin \left(10 \cdot \pi / T_{4}\right) /\left(10 \cdot \pi / T_{4}\right)\right|$ & 0.0294079989 \\
\hline \multirow[t]{2}{*}{11 th } & $11 \cdot w_{04}$ & 4.3196898986 & $\notin D w$ & $/ /$ & \\
\hline & & & & $\sum 4=\sum 4=A_{84}+A_{94}+A_{104}$ & 0.103884918 \\
\hline
\end{tabular}

and interval borders are:

$$
\begin{aligned}
D w_{\min } & =2.9 \cdot w_{Z} / 6 \\
& =3.036872898470133463847221937,
\end{aligned}
$$

Table 3. Numerical results of various $T$, up to $T=80 \cdot \tau$.

\begin{tabular}{ccc}
\hline $\boldsymbol{T} / \boldsymbol{\tau}$ & $\boldsymbol{N}$ harmonics & Sum \\
\hline 4 & 1 & 0.1591 \\
8 & 2 & 0.1384 \\
12 & 3 & 0.1314 \\
16 & 3 & 0.1039 \\
21 & 4 & 0.0972 \\
25 & 5 & 0.1007 \\
30 & 6 & 0.1052 \\
35 & 7 & 0.1084 \\
40 & 8 & 0.1041 \\
50 & 10 & 0.1034 \\
60 & 11 & 0.0965 \\
70 & 15 & 0.1065 \\
80 & 16 & 0.1058 \\
\hline
\end{tabular}

Table 2. Numerical results of $T_{4}=16 . \tau$. 
and:

$$
\begin{aligned}
D w_{\max } & =4.1 \cdot w_{Z} / 6 \\
& =4.293509959906050759232279290 .
\end{aligned}
$$

It is readily seen that the 29 th harmonic:

$$
29 \cdot w_{011}=
$$$$
3.0368728984701334638472219371=D w_{\min },
$$

and the 41th harmonic:

$$
\begin{aligned}
& 41 \cdot w_{011}= \\
& 4.2935099599060507592322792904=D w_{\max },
\end{aligned}
$$

coincide with the borders of interval $D w$.

Whether harmonics will fall inside or outside of interval $D w$ depends on the far 22 nd decimal, i.e.,

$$
\begin{aligned}
D w_{\min } / w_{011} & \\
= & 29.000000000000000000000400470474
\end{aligned}
$$

Amplitudes of harmonics are given in Table 4 from which we can get three different sums depending on whether harmonics (coinciding with border nodes) fall into or out of interval $D w$. Hence:

$$
\begin{aligned}
& \sum 11=A_{2911}+A_{3011}+A_{3111}+A_{3211}+A_{3311} \\
& +A_{3411}+A_{3511}+A_{3611}+A_{3711}+A_{3811} \\
& +A_{3911}+A_{4011}+A_{4111}=0.1139554241,(27) \\
& \sum 11 / 1=\sum 11-A_{2911}=0.1029942636, \\
& \sum 11 / 2=\sum 11-A_{4111}=0.1074442745, \\
& \sum 11 / 3=\sum 11-A_{2911}-A_{4111}=0.0964831140 .(27 \mathrm{c})
\end{aligned}
$$

Table 4. Amplitudes for $T_{11}=60 \cdot \tau$.

\begin{tabular}{llll}
\hline Harmonic & Frequency & \multicolumn{2}{c}{ Amplitude } \\
\hline 29th & $29 \cdot w_{011}$ & $A_{2911}$ & 0.0109611605 \\
30th & $30 \cdot w_{011}$ & $A_{3011}$ & 0.0106103295 \\
31st & $31 \cdot w_{011}$ & $A_{3111}$ & 0.0102539888 \\
32sd & $32 \cdot w_{011}$ & $A_{3211}$ & 0.0098926922 \\
33rd & $33 \cdot w_{011}$ & $A_{3311}$ & 0.0095269989 \\
34th & $34 \cdot w_{011}$ & $A_{3411}$ & 0.0091574721 \\
35th & $35 \cdot w_{011}$ & $A_{3511}$ & 0.0087846783 \\
36th & $36 \cdot w_{011}$ & $A_{3611}$ & 0.0084091859 \\
37th & $37 \cdot w_{011}$ & $A_{3711}$ & 0.0080315643 \\
38th & $38 \cdot w_{011}$ & $A_{3811}$ & 0.0076523829 \\
39th & $39 \cdot w_{011}$ & $A_{3911}$ & 0.0072722099 \\
40th & $40 \cdot w_{011}$ & $A_{4011}$ & 0.0068916112 \\
41st & $41 \cdot w_{011}$ & $A_{4111}$ & 0.0065111496 \\
\hline
\end{tabular}

To view harmonic disposition for $T=50 \cdot \tau$, Figure 5 is presented.

Further calculations are carried out by a short $(\mathrm{C}++)$ program. Results are given in the diagram form in Figure 6.

In repeating shape of diagram one can notice periodic convergence. In this respect, the increment rate is 50 . The diagram starts at $T=50$.

Let us now examine the opposite transition from continuous to line spectrum.

Considering a (finite) sequence of rectangular pulses, with periodic disposition along $t$-axis (Figure 7 ), its spectral function is easily calculated as follows:

$$
\begin{aligned}
S(\omega) & =S_{P}(\omega) \\
& {\left[1+e^{-i \omega T}+e^{-i 2 \omega T}+\cdots+e^{-i(N-1) \omega T}\right] } \\
& =S_{P}(\omega) \sum_{n=0}^{N-1} e^{-i n \omega T},
\end{aligned}
$$

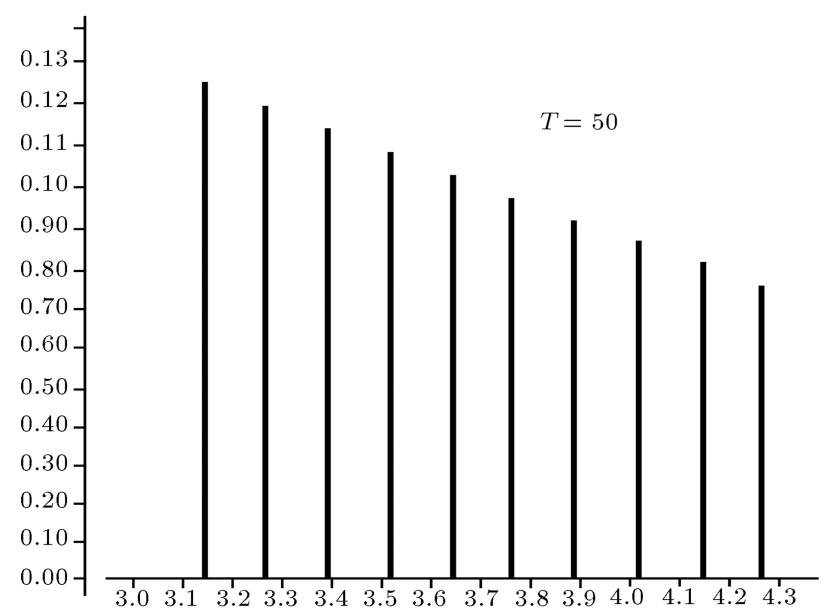

Figure 5. Harmonics' amplitudes for $T=50 \cdot \tau$.

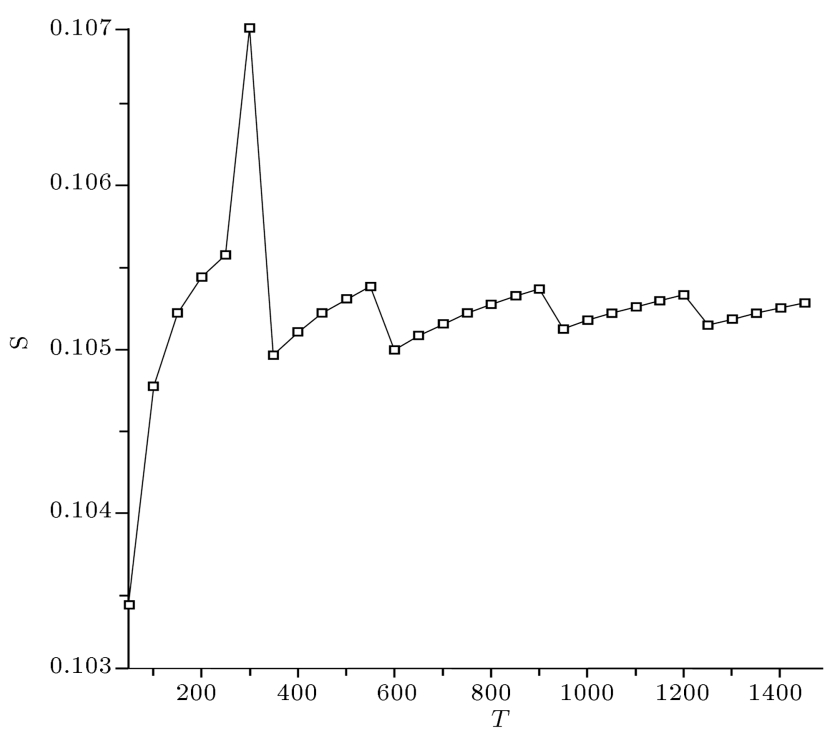

Figure 6. The change of sum as a function of $T$. 

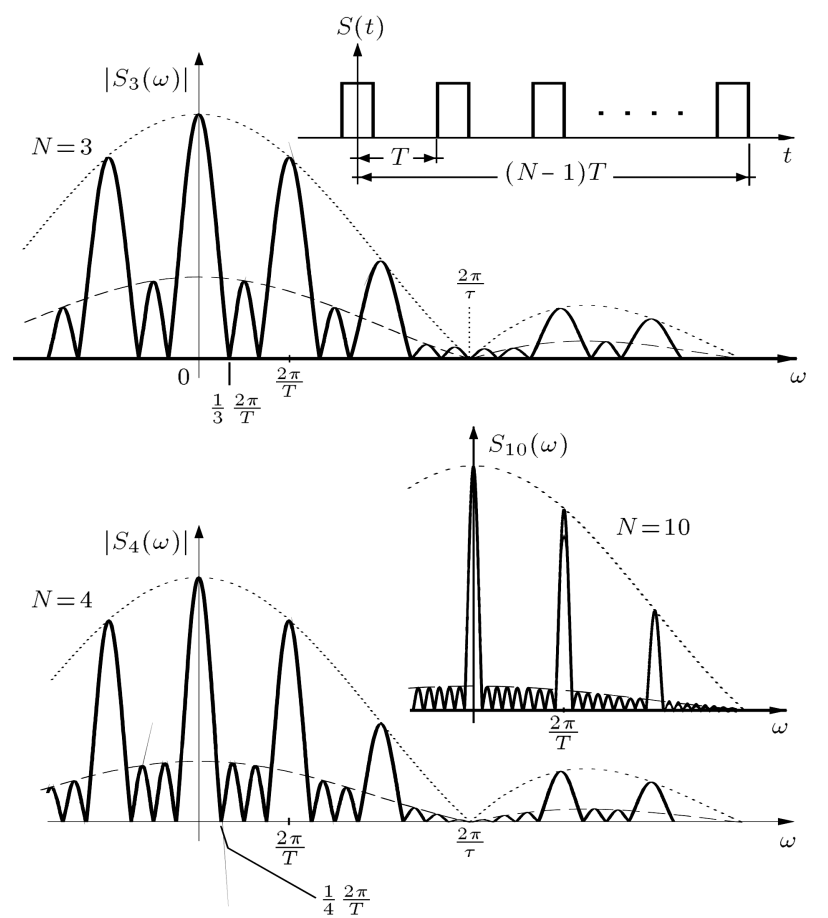

Figure 7. Shape of spectral characteristic of three various rectangular pulse sequences with 3,4 , and 10 pulses. It is readily seen that the tendency of spectral peak rises to infinity and its width to infinitesimal.

where the shift theorem of the FT is used.

The sum of geometric progression with $N$ terms in Eq. (28) is as follows:

$$
\Sigma_{N}=\frac{1-e^{-i N \omega T}}{1-e^{-i \omega T}}=\frac{\sin N \omega T / 2}{\sin \omega T / 2} e^{-i \omega(N-1) T / 2}
$$

and, finally:

$$
S(\omega)=S_{P}(\omega) \frac{\sin N \omega T / 2}{\sin \omega T / 2} e^{-i \omega(N-1) T / 2},
$$

where $N$ is the number of pulses in the sequence, $T$ is the pulse period, and $S_{P}(\omega)$ is the spectral characteristic of the single rectangular pulse.

When the number of pulses in the sequence rises, the change of continuous spectrum shape shows the tendency of localization (Figure 7) ([1], pp. 139-143), $[5,6])$, and rising peaks at harmonic frequencies appear in the places of lines in the discrete spectrum.

To represent the result of this limit process mathematically, Dirac's function is used as follows:

$$
\delta(x)=\left\{\begin{array}{ll}
\rightarrow \infty & x \rightarrow 0 \\
=0 & x \neq 0
\end{array} \quad \int_{-\infty}^{+\infty} \delta(x) d x=1\right.
$$

Herein, we simply cannot help admiring the mathematical elegance of Eq. (31), where infinity and infinitesimal are combined.
Now, two different descriptions of the same entity, i.e., spectral line, are available. This ambiguity reminds us not to confuse the (formal) representation of the entity with the entity itself.

\section{Seismic signal in the periodic form}

If the ever-lasting seismic noise [7] is excluded, the seismic signal can be considered as a fluctuating pulse with a very complicated waveform, as an aperiodic random signal. Depending on its source, there are several categories of seismic signals [8-10]. The propagation of seismic waves through the Earth strongly adds to intrinsic waveform complexity $[9,11]$. Accordingly, the spectrum of the seismic signal (i.e., what is recorded by seismograph in-situ) is inevitably continuous, in which several peaks can be identified that are essential for the interaction between structures and seismic dynamic load.

The seismic waveform from the source (hypocenter) is distorted by dispersion, refraction, multipath propagation of seismic waves, etc., while traveling through a quite inhomogeneous medium. Therefore, at a distance, the processes in the source cannot be easily analyzed. Multiple seismic echoes further complicate the situation, although the distortion within a waveform packet is smaller than that between them $[12,13]$.

On the other hand, seismic perturbation in-situ recorded by seismographs $[14,15]$ is what actually acts on structures, causing damage and casualties and bearing the highest practical importance.

The structure, made up of mutually connected reinforced concrete frames, reacts to seismic perturbations by vibrations composed of so-called tones, i.e., low-frequency harmonics, that stem from dynamic properties of multi-story frame configuration [16-18]. Typical values of a basic oscillating period for multiframe and multi-story RC structures range from 0.15 to $0.5[19]$.

The correspondence between harmonics in the seismic signal and "hidden" harmonics in the structure may clearly reveal the resonance of the interactions. Herein, neither repeated (non-overlapped seismic echoes) nor multiple seismic influences are taken into account [20].

It is recommended to apply the proposed analysis to seismic pulse, of whatever complicated waveform type it might be, and assume an aperiodic shape as periodic one in order to present its spectral content in the spectral line form.

An important question concerning 'how to cut out the most significant part of pulse waveform and how to sequence such pulses into a periodic signal' arises so that noticeable spectral effects of this operation can be minimized; in other words, pulses should be 
tailored without discontinuities. A similar problem in digital signal processing is regularly solved by windowing $[21,22]$. Windowing often indicates edge distortions and carries subjective influence through decisions. Repeating pulses are simply tailored by taking a packet waveform as a basic period and relying on the similarity of edge fluctuations in amplitude and slope [22,23]. Obviously, a subjective factor, similar to that in windowing, is involved in this process; however, this study, driven by its predilections, considers this factor to be a much more natural approach based on variations in the waveform and, broadly, researchers' experience, very desirable in seismic analysis [3]. We hope that this approach will be helpful as an addition to the seismic-analysis-related methods [24,25].

Let us explain our approach through Figure 8 [24]. Point $S$ in Figure 8 indicates the emergence of an earthquake, which develops itself through strong fluctuations of the main portion of the pulse, followed by relaxations and ceasing. We state that the main portion of the seismic pulse (roughly, from 1150 to 1350 seconds in Figure 8) imposes strong impact on structures.

The subsequent relaxation portion of the seismic pulse (from about 1375 to 1460 seconds) imposes, in relative terms, the same level of impact on structures since they are already damaged and weakened by the preceding strong impact. To incorporate all these significant parts of seismic impulse into our analysis, it is estimated that a level $10 \%$ of extremal amplitude may be considered as the best. Therefore, the cutting points are determined (zoomed in Figure 8). This basic criterion is combined with amplitudes of peaks that precede and follow the cutting points [12].

Now, we have a seismic pulse without beginning and ending tails, especially ending, which is an important picture of earthquake ceasing. However, the crucial portion of seismic pulse, responsible for destroying effect, is included. It is now possible to calculate a line spectrum by creating a periodic signal from this pulse or simply by assuming it as a periodic signal. The only question to respond is how to choose the value of period, i.e., the value of the fundamental harmonic depending on the required density of the spectrum.

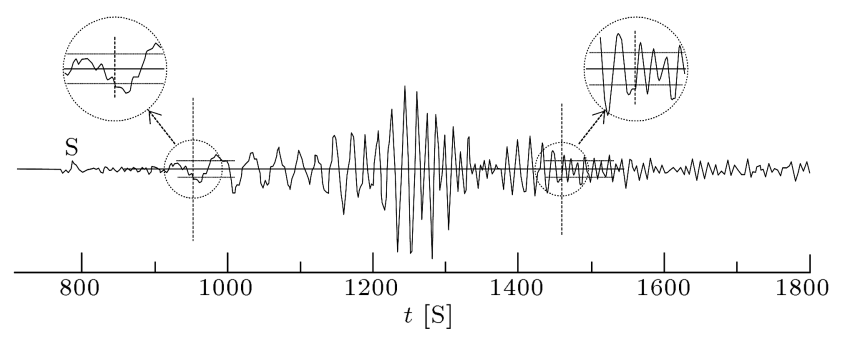

Figure 8. Seismic pulse of Haiti earthquake, January 12, 2010 at 21:53:09 UTC, recorded at Lac du Bonnet, Manitoba, transverse displacement, Love waves.

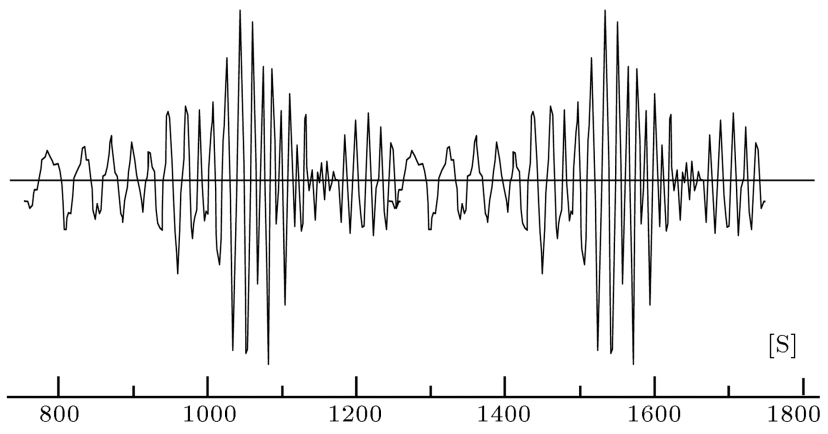

[S]

Figure 9. A periodic seismic signal formed by repeating and seamless tailoring of the impulse in Figure 8.

A periodic seismic signal composed of such pulses is given in Figure 9. It resembles a double-side-band amplitude modulated signal ([6], pp. 72-80) composed of two prominent frequencies: seismic fluctuations as a "carrier" and repeating rate of the whole pulse as a fundamental harmonic.

\section{Conclusion}

Among other techniques, such as those of Havskov and Aguacil [15] and Ćosić et al. [25], spectral analysis is a widely used tool for seismogram interpretation. There are many categories of spectrum users in the seismic community [3]. Spectrum tools are closely related to dedicated computer software, often yielding readymade results and hiding calculation details. Users usually communicate with this software over Graphical User Interface (GUI). Despite tremendous advances in GUI technology, one cannot claim that it is always optimal (user-friendly at high extent).

Hence, a researcher/user encounters twofold opacity of such software: hidden program codes and conditions of their use, represented indirectly through GUI and written by some seismic experts and/or designers of GUI.

In addition, the frequent use of spectral tools in the explication of seismic matters inevitably is normalized and is followed sometimes by self-confidence, feeling of easiness, and albeit insufficient attention. Habit often disrupts carefulness. As humans are dominantly visual beings, they often show a subconscious tendency toward treating the GUI of tools as tools per se. Hence, it is recommended that psychological analysis be taken worthy of further study and research in this regard.

Researchers are not immune to such effects, particularly when unusual cases are analyzed and interpreted.

Because of all these circumstances, it is important to make contributions for a better understanding of subjects and tools of our research. We attempted to highlight a new aspect of the relation between line and 
continuous spectra through the transition process, done in two directions, to clarify the fundamental notions of spectral line, from both sides, and spectral density.

Although spectral analysis may seem as an outdated issue belonging to the classical literature, we state that our analysis, presented in this paper, brings into attention a valuable aspect of spectrum behavior, either of line or continuous cases. As a shortcoming, it was impossible to survey each item of available literature and trust them, or to compare our approach to possible existing similar ones, because this issue relates to the very fundamental concept of Fourier tools that was studied a long time ago in very broad terms. Nevertheless, our theoretical and numerical tracing of beginning stages of the transition process from line toward continuous spectrum has revealed interesting behavior, involving invariance through convergence.

Similar processes associated with spectral lines from continuous spectrum emerge. A careful consideration helps clarify the essence of interpretation of spectral terms when using such mathematical tools.

Thus, the idea of using periodization of fragments of seismic signal, instead of windowing technique, to represent important parts of seismic waveform in terms of the line spectrum was used to describe spectral content clearly, thus facilitating the efforts to create a correspondence between a structure's tones to directly estimate possible resonances that can have high significance in considering seismic influence on structures.

Of note, matching does not merely mean coincidence of (seismic) harmonics and (structure) tones, but rather considering the distances between close and corresponding ones, where another direction of further research appears so as to create a resonance between intensity and distance.

Determining the criteria required for choosing and extracting portions of seismic signal along with their influence on structures remains a mainstream research. Herein, this study offers a starting point for defining some new attributes of seismic signals.

\section{References}

1. Babić, R., Signal Analysis I, Accademic Mind, Belgrade, Serbia (2000) (in Serbian).

2. Babić, R. Signal Analysis II: Probability, Random Signals, Solved Problems, pp. 111-158, Accademic Mind, Belgrade, Serbia (2008) (in Serbian).

3. Bormann, P. "Aim and scope of the IASPEI new manual of seismological observatory practice", In New Manual of Seismological Observatory Practice, pp. 5-22, revised version, Deutschen GeoForschungsZentrums-GFZ, Germany (2002), epublished (2009).

4. Yarlagadda, R.K., Analog and Digital Signals and
Systems, pp. 109-129, Springer/Dordrecht Heidelberg, New York/London, USA/UK (2010).

5. Harkevich, A.A., Spectrums and Analysis, pp. 27-30, GIFML, Moscow, Russia (1962) (in Russian).

6. Gonorovsky, I.S., Radio Circuits and Signals, pp. 39-52, MIR Publishers, Moscow, Russia (1981) (in Russian).

7. Bormann, P. "Seismic signals and noise", In New Manual of Seismological Observatory Practice, pp. 187-219, revised version, Deutschen GeoForschungsZentrumsGFZ, Germany (2002), e-published (2009).

8. Bormann, P., Baumbach, M., Bock, G., Grosser, H., Choy, G.L., and Boatwright, J. "Seismic sources and source parameters", In New Manual of Seismological Observatory Practice, pp. 93-186, revised version, Deutschen GeoForschungsZentrums-GFZ, Germany (2002), e-published (2009).

9. Lee, W.H.K., Jennings, P., Kisslinger, C., and Kanamori, H., International Handbook of Earthquake \& Engineering Seismology, Part A, pp. 209-235, Academic Press, Cambridge, USA (2002).

10. Kostrov, B.V. and Das, S., Principles of Earthquake Source Mechanics, pp. 17-42, Cambridge University Press, Cambridge, USA (1988).

11. Bormann, P., Engdahl, B., and Kind, R. "Seismic Wave Propagation and Earth models" In New Manual of Seismological Observatory Practice, pp. 23-92, revised version, Deutschen GeoForschungsZentrumsGFZ, Germany (2002), e-published (2009).

12. Bormann, P., Klinge, K., and Wendt, S. "Data analysis and seismogram interpretation", In New Manual of Seismological Observatory Practice, pp. 527-627, revised version, Deutschen GeoForschungsZentrumsGFZ, Germany (2002), e-published (2009).

13. Polyakov, S.V., Design of Earthquake Resistant Structures, pp. 72-108, Vyshaya Schola, 2nd ed., Moscow, Russia (1983) (in Russian).

14. Wielandt, E. "Seismic sensors and their calibration" In New Manual of Seismological Observatory Practice, pp. 221-296, revised version, Deutschen GeoForschungsZentrums- GFZ, Germany (2002), epublished (2009).

15. Havskov, J. and Alguacil, G., Instrumentation in Earthquake Seismology, pp. 149-196, Springer, New York, USA (2015).

16. Meyers, R.A., Extreme Environmental Events: Complexity in Forecasting and Early Warning, pp. 150-218, Springer Science \& Business Media, New York, USA (2010). 
17. Paulay, T. and Priestley, M.J.N., Seismic Design of Reinforced Concrete and Masonry Buildings, pp. 1-94, John Wiley \& Sons, New York, USA (1992).

18. Clough, R.W. and Penzien, J., Dynamics of Structures, 3rd Ed., pp. 669-729, Computers \& Structures, Inc., Berkeley, USA (1995).

19. Folić, R. and Babić, L. "Analysis of RC frame with masonry and panel infil behaviour in seismic regions", 10th International Scientific Conference VSU, Sofia, Bulgaria (2010).

20. Liolios, A. and Hatzigeorgiu, L. "Effects of multiple earthquakes to the seismic response of structures", DIMK, Building Materials and Structures, 55(4), pp. 3-15 (2012).

21. Stein, J.Y., Digital Signal Processing: A Computer Science Perspective, pp. 495-530, John Wiley \& Sons, Inc., New York, USA (2000).

22. Rabiner, L.R. and Gold, B., Theory and Application of Digital Signal Processing, pp. 103-124, Mir, Moscow, Russia (1978) (trans. to Russian).

23. Havskov, J. and Ottemoller, L., Routine Data Processing in Earthquake Seismology: With Sample Data, Exercises and Software, pp. 83-99, Springer Science \& Business Media, New York, USA (2010).

24. Seismic pulse of Haiti earthquake, URL: https://seislog.wordpress.com/2010/01/13/haiti/

25. Ćosić, M., Folić, R., and Brčić, S. "An overview of modern seismic analyzes with different ways of damping introduction", DIMK, Building Materials and Structures, 60(1), pp. 3-31 (2017).

\section{Biographies}

Ranko Babić obtained BS degree in Electrical Engineering from University of Pristina, Serbia in 1981, MSEE in Electronic Engineering from University of Nis, Serbia in 1987, and a PhD (coding algorithms in OCR) degree in 1993. He is currently a Professor of Communications and Signals at the Department of E.E. at the School of Technical Sciences, University of Pristina and, also, at the School of Railway Applied Studies, Belgrade. His research interests include methods of signal analysis, current seismic signals, optoelectronic gravity and seismic sensors, aspects of energy effectiveness of high-speed trains.

Lidija Babić obtained a BS degree in Civil Engineering from University of Pristina, Serbia in 1995, MSCE (Rheology of Materials) degree in 2006, and a PhD (Seismic Engineering) degree in 2017. Her current research includes seismic behaviour of brick infilled RC frames.

Branimir Jakšić received $\mathrm{BSc}$ and MSc degrees in Electrical Engineering from the Faculty of Technical Sciences in Kosovska Mitrovica, University of Pristina, Serbia and PhD degree in Electrical Engineering from Faculty of Electronic Engineering, University of Nis, Serbia in 2015. He is an Assistant Professor at the Faculty of Technical Sciences in Kosovska Mitrovica. His areas of research include statistical communication theory and optical telecommunications. He has authored over 50 scientific papers on the above subject. 